\title{
Selective Inhibition of Paraffin Deposition under High Flow Rate as a Function of the Crude Oil Paraffin Type and Content by Fluorescence Depolarization: Polypropylene and High-Density Polyethylene
}

\author{
Cristina M. Quintella,* Angelo M. V. Lima, and Eduardo B. Silva \\ Instituto de Química, Universidade Federal da Bahia, Campus de Ondina, \\ Salvador, BA, Brazil, CEP: 40.170-290 \\ Received: October 17, 2005; In Final Form: February 6, 2006
}

\begin{abstract}
High-density polyethylene (HDPE) and polypropylene (PP) were studied to identify tailor-made materials for walls of pipelines and ducts for crude paraffinic oil that inhibit paraffin depositions. The interfacial interaction was investigated from 50 to $70{ }^{\circ} \mathrm{C}$ and as a function of the $n-\mathrm{C}_{36} \mathrm{H}_{74}$ paraffin concentration added to the paraffinic crude oil. The static and the dynamic interfacial tensions were observed, respectively, by traditional contact angle measurements and by fluorescence depolarization of the natural fluorescent probes of the crude oil, flowing at a high rate. The static interaction showed a low dependence on the $n$-paraffin content for both surfaces. For PP, it decreased slightly as the $n$-paraffin concentration increased, evidencing an increase in the liquid-liquid interaction. The dynamic interfacial tension with PP clearly decreased as temperature and $n$-paraffin concentration increased, the latter effect being attributed to the PP methyl groups hindering the interaction between the $n$-paraffin and the PP main chain. For the flow on HDPE, the interaction proved to be highly dependent on the $n$-paraffin concentration and temperature. It increased as $n$-paraffin was added and temperature decreased. The former effect is attributed to the alignment of the $n$-paraffin chains within the high rate flowing liquid and the similar molecular geometry of the $n$-paraffin and the linear polymer surface (almost without branches), which increases the number of sites available for interaction. PP proved to be more suitable for transportation of crude oil rich in paraffins with more than 36 carbon atoms, while HDPE was more suitable for those with smaller paraffinic chains.
\end{abstract}

\section{Introduction}

A recent trend in crude oil transport is the use of ducts made of plastics, amorphous polymers, and fiber-reinforced plastic polymers. They may be used either as coatings or linings for metallic ducts or as massive ducts. Although they cannot stand high temperatures and their low mechanical resistance and creep life is a concern, ${ }^{1}$ they have the advantages of impeding the formation of paraffin deposits ${ }^{2-4}$ and a much lower corrosion rate than unlined metallic ducts. They can be used to recuperate condemned metallic ducts by insertion of a plastic layer as the lining, thus reducing the costs of welding and equipment maintenance.

In addition, all over the world, as crude oil exploitation evolves, there is a trend to produce heavier oils, due to the preferential production of the more volatile fractions in the first years. Heavy oils have a high content of wax or paraffin, as well as asphaltenes. Although the technology to process these heavy oils by the refineries is well-known, their pumping and duct transportation is hindered by precipitation of asphaltenes and paraffins that increase the viscosity and may yield deposits ${ }^{4,5}$ along the inner walls, requiring higher pumping capacity and increasing the probability of equipment failures and leakage. ${ }^{6}$ Paraffin crystallization depends on the thermodynamic and fluid dynamic conditions, the crude oil paraffin content, and the paraffins molecular structure. ${ }^{7}$ The increase in paraffin intermolecular alignment leads to their nucleation and, consequently, to the beginning of crystal formation. ${ }^{8,9}$

* To whom correspondence should be addressed. E-mail: cristina@ufba.br. Address: LabLaser, Instituto de Química, Universidade Federal da Bahia, Campus de Ondina, 40.170-290, Salvador-BA, Brazil. Tel: 55-71-99647876. Fax: $55-71-32355166$.
Nowadays, most of the ducts for production and transport are metallic. Nevertheless, in the mid-to-long term, most of the production tubing, transportation ducts, and pipelines will need to be customized to the operational conditions and to the types of fluids they transport to decrease the financial losses and to preserve the environment. These tailor-made ducts must reduce the chemical affinity with the fluids, that is, decrease the wetting efficiency that leads to degradation like corrosion and the formation of asphaltenic and paraffinic deposits.

To develop these tailor-made ducts, it is necessary to know the wetting efficiency, which increases the adhesion capacity and, consequently, increases the drag intensity. The wetting increases when the interfacial tension between the pipeline walls and the fluids increases. Because the interfacial interactions take place at the boundary layers, where molecular effects overtake hydrodynamic ones, the flow rate should be a variable with high significance since high flow rates also increase the molecular effects over the fluid dynamic ones. ${ }^{10}$

In the last 50 years, the interfacial tension has been determined mostly under static conditions or at very low flow rates using the contact angle technique $(\theta \mathrm{c}) \cdot{ }^{10}$ For a given liquid, the interfacial tension is proportional to the cosine of $\theta \mathrm{c}$. It is known that $\theta \mathrm{c}$ depends on the surface roughness, ${ }^{10}$ the chemical nature of both the solid surface and the liquid, ${ }^{4,11-14}$ and the relative orientation of the surface chemical groups. ${ }^{15}$ Zhang et al. ${ }^{16}$ studied coatings of polyurethane, an epoxy resin, two polyvinylidene fluoride polymers, two silicone rubbers, and a methyl acrylate-stylene copolymer, evaluating the dynamic contact angle at very low flow rates and the corresponding hysteresis factor of the coatings against asphalt, ozocerite, and 
crude oil. They found that the last five coatings were better for drag reduction and paraffin deposition prevention, although they did not specify for which paraffin type or their chain lengths and branching degrees.

For high flow rates, the depolarization of the fluorescence excited by laser in induced liquid flows (PLF-FI) has been used in the past decade to study the dynamic interfacial tension between solid walls and liquids. PLF-FI has several advantages over $\theta$ c: (1) it can be used with liquids at high velocities; (2) when using a transparent window, it is a noninvasive and a nondestructive test (NDT); (3) it can be remotely applied; (4) it is more sensitive to wall chemical constitution than static $\theta \mathrm{c} ;{ }^{17}$ (5) it reflects processes at a molecular level, when the preliminary orientation that leads to crystal formation starts to take place, thus having high potential as a remote method for indicating the beginning of paraffin deposits.

PLF-FI showed that the wetting of surfaces by liquids at high flow rates is sensitive to the chemical nature of both the walls and the liquid ${ }^{12}$ and to the surface oxygen content. ${ }^{17}$ It also proved to be sensitive to the branching degree of polyethylene surfaces, although the static interfacial tension evaluated by traditional $\theta \mathrm{c}$ was not. ${ }^{17}$ This sensitivity was associated with the wetting due to temporary intermolecular interactions between the flowing liquid and the wall surface. A pronounced effect of the wall's chemical constitution on the flow induced intermolecular alignment ${ }^{18}$ was observed for microchannels $10 \mu \mathrm{m}$ wide.

Recently, ${ }^{4}$ we showed that the natural fluorescence of crude oil can be used, without addition of fluorescence probes, to monitor the intermolecular alignment of paraffins during flow and their interaction with pipeline walls with different chemical constitutions. The wettability of an $80 \%(\mathrm{v} / \mathrm{v})$ crude oil in heptane solution on surfaces of polypropylene (PP), high-density polyethylene (HDPE), and vinyl acetate copolymer with $28 \%$ oxygen content (EVA28), as well as the potential for paraffin deposition inhibition, was studied at $25^{\circ} \mathrm{C}$, and PP was found to be more suitable for inhibiting wax deposition. This study also confirmed the emphasis of the molecular effects over the fluid effects when the liquid is at a high flow rate.

The aim of this paper is to define which polymer surface is more suitable for hindering paraffin deposition as a function of the paraffinic content of the crude oil and at temperatures near, but below, the cloud point. For this purpose, we evaluated the static and dynamic interfacial tensions between crude oil and two polymer surfaces by $\theta \mathrm{c}$ and PLF-FI, respectively. The study comprises three different temperatures and the addition of four different concentrations of a normal paraffin with a fusion point just above the highest temperature studied.

As far as we are aware, this is the first time that a wetting study is reported that considers the type of polymer wall and the type and crude oil paraffinic content, as well as the operational temperature.

\section{Experimental Section}

2.1. Materials. The crude oil was from Bacia do Recôncavo Baiano and consisted of a crude dead oil, that is, as produced at the well without the volatile fraction. It was brown and had a composition typical of algal organic matter from a paleolithic lagoon environment with $14.7 \%(\mathrm{w} / \mathrm{w})$ paraffin content, $0.20 \%$ (w/w) emulsed water, sediments below traces, $0.8727 \mathrm{~g} \mathrm{~cm}^{-3}$ density at $15.6{ }^{\circ} \mathrm{C}, 30.64{ }^{\circ} \mathrm{API}, 39.00{ }^{\circ} \mathrm{C}$ average pour point, and average cloud point at $49.22^{\circ} \mathrm{C}$.

Time-resolved spectroscopy ${ }^{4}$ showed that the intrinsic fluorescent probes of the crude oil excited at $532 \mathrm{~nm}$ has a

\begin{tabular}{|c|c|}
\hline$-\left(\mathrm{H}_{2} \mathrm{C}-\mathrm{CH}_{2}\right)_{\mathrm{n}}$ & $\begin{array}{c}\mathrm{CH}_{3} \\
\text { HDPE }\end{array}$ \\
H. & PP \\
\hline
\end{tabular}

Figure 1. Scheme of the PP and HDPE polymer structures.

TABLE 1: API Degree as a Function of the $n-\mathrm{C}_{36} \mathrm{H}_{74}$ Paraffin Added

\begin{tabular}{ccc}
\hline mass of added $n-\mathrm{C}_{36} \mathrm{H}_{74}(\mathrm{~g})$ & $\%(\mathrm{w} / \mathrm{w})$ & ${ }^{\circ} \mathrm{API}$ \\
\hline 0 & 0 & 39.1 \\
1.4 & 0.6 & 33.0 \\
2.8 & 1.2 & 30.1 \\
4.3 & 1.8 & 29.6
\end{tabular}

fluorescent emission with a maximum at $582 \mathrm{~nm}$, width at halfheight of $73 \mathrm{~nm}$, and an excited lifetime of $3.8 \mathrm{~ns}$. The natural crude oil fluorophores consist mainly of polycyclic aromatic hydrocarbons (PAHs) with lateral alkyl chains and, as the number of rings increases, the alkyl chain length increases proportionally. ${ }^{7}$ At this excitation wavelength, the number of rings should be at least over three. Gas chromatography-mass spectrometry (CG-MS) allowed the identification of peaks which were attributed to choronanes.

The ramification degree was 0.65 , as obtained by Fourier transform infrared spectroscopy using the $\mathrm{CH}_{3} / \mathrm{CH}_{2}$ areas of the respective paraffin symmetric angular deformations.

Differential scanning calorimetry showed that the crude oil had a wide region of cloud points from 37 to $70{ }^{\circ} \mathrm{C}$, which correspond, respectively, to the melting of paraffins with average carbon numbers from 21 to 32 . Thus, the increase in temperature from 50 to $70{ }^{\circ} \mathrm{C}$ in this study should increase the concentration of melted paraffins with up to 32 carbons atoms.

The paraffinic content was increased by adding the $n$-paraffin $n$-hexatriacontane $\left(n-\mathrm{C}_{36} \mathrm{H}_{74}\right)$ (Aldrich, 630-06-8) with a fusion point of $75^{\circ} \mathrm{C}$ (Table 1). Before the paraffin addition, the crude oil was heated to $70{ }^{\circ} \mathrm{C}$. The $n$-paraffin was then added, and the oil was mixed until complete dissolution.

The temperature was controlled by a Nova Ética Model 521/ 10 thermostatic bath with $0.1{ }^{\circ} \mathrm{C}$ precision. The water was pumped through a jacket surrounding the entire crude oil flowing system, giving an error margin of $0.5^{\circ} \mathrm{C}$.

The wall surfaces consisted of two aliphatic long chain polymers (Figure 1), high-density polyethylene (HDPE, IA-59 Politeno), and polypropylene (PP, Moltec). The polymer surfaces were made in the same setup by ASTM D 4703 to obtain homogeneous, isotropic plaques, which yields $40-60 \%$ crystallinity and isotactic PP. Differential scanning calorimetry showed that the HDPE had $49 \%$ crystallinity and that the PP had 59\% crystallinity and was isotactic. The HDPE had a weight-average molecular weight $\left(M_{\mathrm{w}}\right)$ of 44113 , average molecular weight $\left(M_{\mathrm{n}}\right)$ of 13353 , and molecular weight distribution PD $=\left(M_{\mathrm{w}} /\right.$ $M_{\mathrm{n}}$ ) of 3.30. PP had an $M_{\mathrm{w}}$ of $395996, M_{\mathrm{n}}$ of 44627 , and PD of 8.87 .

The polymer surfaces had similar roughness that varied between 80 and $100 \mathrm{~nm}$, as determined by perfilometry. They measured $2 \mathrm{~cm} \times 4 \mathrm{~cm}$ with a thickness of $0.5 \mathrm{~cm}$. All surfaces were cleaned with water, then immersed for $10 \mathrm{~min}$ in ethanol and dried at $25^{\circ} \mathrm{C}$ for at least $15 \mathrm{~min}$ before each experiment.

The static interaction was measured by $\theta$ c. A drop of $15 \times$ $10^{-6} \mathrm{~L}$ was formed with a micropipet on the substrates, and its image was remotely acquired by a Hitachi VM-E230A video camera, with $36 \mathrm{X}$ zoom, connected to a PC. The software Image Pro Plus was used to process the images. The final angle was the average of at least five measurements. 

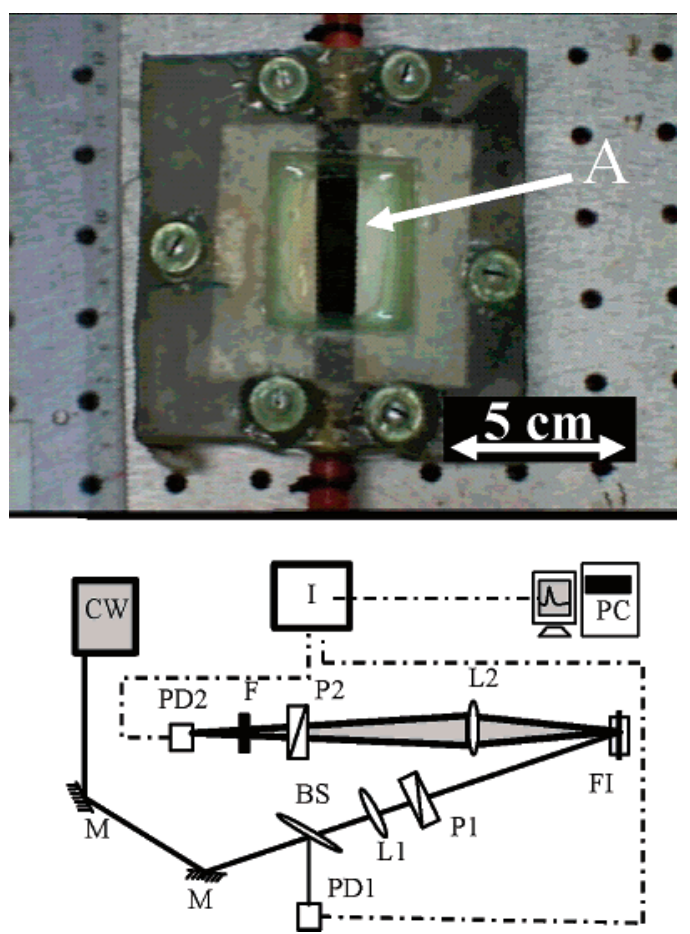

Figure 2. (top) Photo of the flow cell for PLF-FI. (bottom) Experimental setup for polarized laser induced fluorescence within liquid induced flows (PLF-FI) to detect fluorescence depolarization: CW, laser; M, mirrors; L1, L2, lenses; P1, P2, Glan-Thompson Polarizers; PD1, PD2, photodiodes; I, interface; PC, personal computer; FI, liquid flow; F, color filter; BS, beam splitter. (Reproduced with permission from Química Nova, 2005, Vol. 28, No. 2, 227-339. Copyright Sociedade Brasileira de Química.)

2.2. Flow Cell. An in-house developed cell similar to that reported before ${ }^{19}$ (Figure 2 top) was used in order to emphasize the boundary layer where the wall-liquid flow interaction is strongest.

It consisted of two juxtaposed plates with $1 \mathrm{~mm}$ between them, $1.0 \mathrm{~cm}$ width, and $7.0 \mathrm{~cm}$ in length. The front plate was a polycarbonate window, and the bottom plate was the material to be evaluated. The cell body was made of polyester resin. The liquid entered the cell at its top and exited at its bottom through $4.0 \mathrm{~mm}$ diameter tubes. The crude oil velocity was fixed at $47 \pm 5 \mathrm{~cm} \mathrm{~s}^{-1}$. This high velocity was chosen in order to emphasize the molecular effects that become predominant at high velocities over the fluid dynamics effects that predominate at low velocities. ${ }^{10}$ The flow within the cells is considered to be laminar with a Reynolds number below 542 .

2.3. Depolarization of the Fluorescence Induced by Laser in Induced Liquid Flows (PLF-FI). Fluorescence depolarization (PLF) is a technique known for many years to be a sensitive probe of molecular alignment in liquid flows. ${ }^{20}$ Kenyon et al. ${ }^{21,22}$ applied PLF to free liquid laminar jets at high velocity for the first time and found that the intermolecular alignment along the flow is between 1 and 3 orders of magnitude greater than that achieved by conventional flow or electric field alignment techniques. ${ }^{23}$ For liquids flowing at a high flow rate through and out of a thin slit, it proved possible ${ }^{24,25}$ to visualize directly the boundary layer at a molecular level, to infer the macroscopic velocity gradient, to generate the velocity profiles throughout the flow, and to discuss macroscopic fluid dynamic concepts in terms of interactions between the molecules that comprise the flowing liquid.

PLF-FI does not resolve the spectral distribution of the fluorescence but rather its polarization components. PLF-FI consists basically of the irradiation of an appropriate fluorescent molecular probe within a liquid flow by a vertically polarized laser and the detection of the polarization components of the probe fluorescent emission.

For thin flows, the depolarization of the fluorescence, compared with the laser polarization, can be interpreted as a bidimensional phenomenon in terms of polarization $(P)^{21-23}$

$$
P=\frac{I_{\|}-I_{\perp}}{I_{\|}+I_{\perp}}
$$

where $I_{\|}$and $I_{\perp}$ are, respectively, the fluorescence components parallel and perpendicular to the axis defined by the direction of the laser polarization.

The polarized laser preferentially photoselects the fluorescent probes with their molecular transition dipole moment oriented along the flow, with the absorption probability being proportional to the cosine squared of the angle between the laser electrical field and the molecular transition dipole. The flow velocity gradient induces an inner stress that changes the chemical environment, that is, the intermolecular interactions increase the local anisotropy, hindering the rotation of the fluorescent probe. ${ }^{23}$ This increases the probability of the probe still being aligned along the flow when it suffers fluorescent decay, thus increasing the net polarization of the fluorescence emission.

When the interaction between the liquid flow and the solid surface increases, the liquid intermolecular network that was previously aligned vertically by the vertical flow, the additional interactions along the horizontal partially misalign the molecular domains and thus the fluorescent probes, decreasing $\mathrm{I}_{\|}$and consequently $\mathrm{P}$.

The experimental setup for PLF-FI has been described before. ${ }^{17,26}$ Briefly, a Coherent Inova60 argon laser (Figure 2, bottom) in multimode, multiline, and $100 \mathrm{~mW}$ is reflected by two mirrors (M1 and M2) through a biconvex borosilicate lens (L1) of $400 \mathrm{~mm}$ focal length that focuses the laser to a 0.02 $\mathrm{mm}^{2}$ diameter spot on the sample (FI). To monitor the laser intensity fluctuations in real time, $10 \%$ of the laser beam was diverted by a beam splitter (BS) onto a BPW-21 RS-Electronics photodiode (PD1) with a $7.5 \mathrm{~mm}^{2}$ active area, operating as a current to voltage converter with seven optional sensitivity ranges. A vertical Glan-Air polarizer (P1) ensured a $100 \%$ vertically polarized laser beam. The laser beam enters parallel to the vertical of the front window, thus ensuring that only vertically polarized light enters the flow cell. The resultant fluorescence is collected frontally, perpendicular to the front window, thus ensuring that no particular polarization orientation is favored.

Fluorescence was collected within a 0.02 sr solid angle by a biconvex borosilicate lens (L2) of $50 \mathrm{~mm}$ focal length and focused onto a detector consisting of an OPT 202 Burr-Brown photodiode (PD2) with a $5.22 \mathrm{~mm}^{2}$ active area, operated as a current to voltage converter with four optional sensitivity ranges. A $550 \mathrm{~nm}$ cutoff filter (F) blocked the laser radiation. The GlanThompson polarizer (P2) was manually rotated to select either the vertical $\left(I_{\|}\right)$or the horizontal $\left(I_{\perp}\right)$ fluorescence intensities. The outputs of both PD1 and PD2 were captured by an interface (I) connected to a computer (PC). A QBasic program controlled both data acquisition and sample positioning.

The flow cell position was varied uniformly in relation to the horizontal of the laser beam direction, using a two-axis translation frame ${ }^{27}$ with $10 \mu \mathrm{m}$ resolution and repeatability better than $0.1 \%$. Both $I_{\|}$and $I_{\perp}$ were obtained cross-stream at $3.0 \mathrm{~cm}$ 


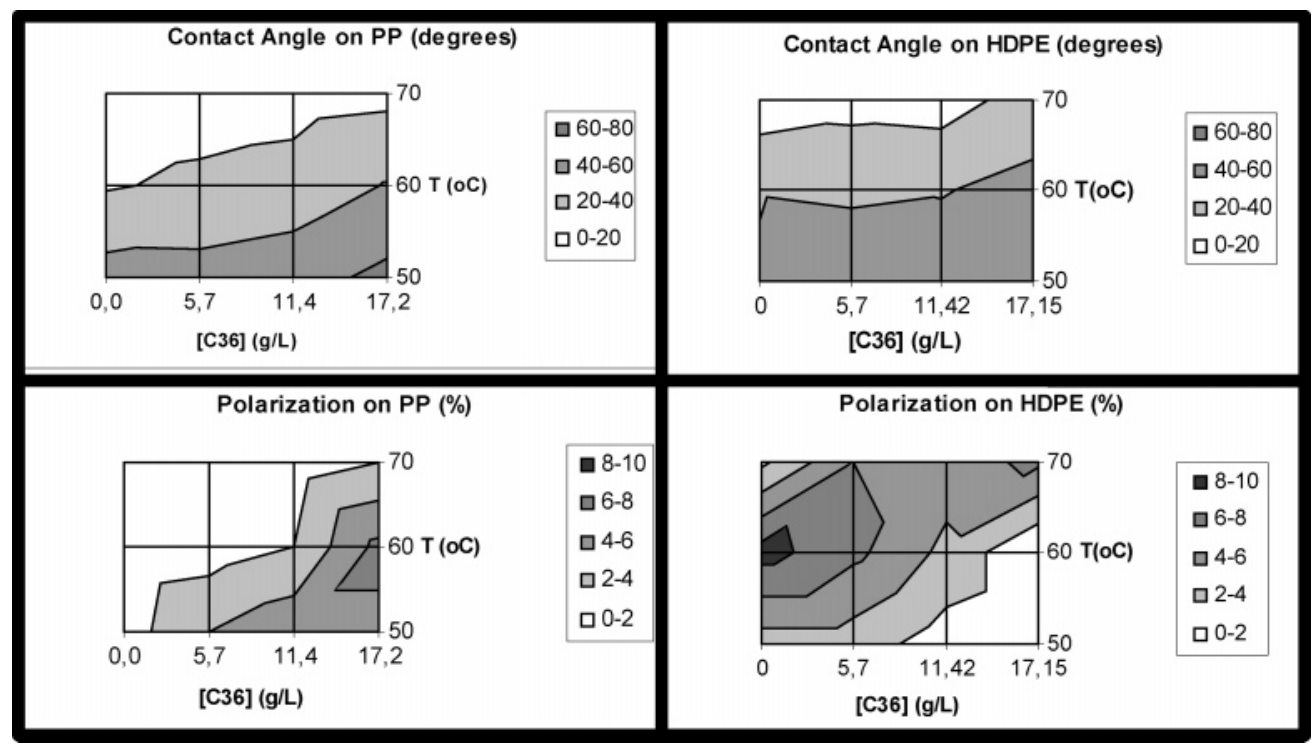

Figure 3. Maps of contact angle (top) and polarization (bottom) as a function of temperature and $n-\mathrm{C}_{36} \mathrm{H}_{74}$ paraffin concentration.

downstream from the cell flow input (part A of Figure 2, top). Polarization profiles acquired further downstream did not change significantly $(<0.2 \%$,). Each profile was acquired three times for the same cell, and their average was used.

Each polarized fluorescence profile (obtained by PD2) was divided by the laser intensity (obtained in real time by PD1) to correct for laser intensity fluctuations.

\section{Results and Discussion}

For each polymer surface, three temperatures and four concentrations of $n$-paraffin were evaluated, for a total of twelve different conditions. These conditions were investigated statically by $\theta \mathrm{c}$ and dynamically by fluorescence depolarization. The data were plotted as maps of $\theta \mathrm{c}$ and polarization as a function of temperature and $n$-paraffin concentration (Figure 3).

3.1. Static interaction: Contact Angle. Because the static interfacial tension is proportional to the cosine of $\theta \mathrm{c}$ and all the angles obtained are below $90^{\circ}$, an increase in the contact angle means a decrease in the interfacial tension. The contact angles (Figure 3, top) had a similar behavior for both HDPE and PP. As the $n-\mathrm{C}_{36} \mathrm{H}_{74}$ concentration increased, there was a slight increase in $\theta \mathrm{c}$ for both surfaces. Thus, the static interfacial tension decreased slightly, showing an increase in the liquidliquid interaction attributed to an increase in the paraffinic crystal content. For PP, $\theta$ c was more sensitive to the $n-\mathrm{C}_{36} \mathrm{H}_{74}$ concentration.

As temperature increased, $\theta \mathrm{c}$ decreased; thus, the static interfacial tension increased with temperature. This was due to less effective liquid-liquid intermolecular interactions as the internal energy increases and crude oil paraffins with longer molecular chains melt, reducing the amount of crystalline nuclei within the liquid phase. At the lower temperature and without $n$-paraffin addition, $\theta \mathrm{c}$ is $42^{\circ}$ for HDPE and $48^{\circ}$ for PP, showing that, under these conditions, the interaction is stronger for HDPE. This is in accord with previous observations ${ }^{4}$ with a $80 \%(\mathrm{v} / \mathrm{v})$ crude oil in heptane solution at $25^{\circ} \mathrm{C}$.

Considering all temperatures and $n$-paraffin concentrations used in this work, the average $\theta \mathrm{c}$ values for HDPE and for PP were, respectively, $35^{\circ}$ and $31^{\circ}$, showing that, on average, the static interfacial tension was higher for PP, regardless of temperature and concentration, over the range studied here.
3.2. Dynamic Interaction (flowing liquid): Fluorescence Depolarization. Figure 3 bottom shows the polarization for the flow on each surface. An increase in polarization corresponds to a decrease in the dynamic interfacial tension. The polarization had a clear dependence on temperature and concentration.

As a general trend, on PP, the polarization decreased with increasing temperature. Thus, the dynamic interfacial tension increased as more natural paraffins of the crude oil melt. The polarization showed a clear increase with $n-\mathrm{C}_{36} \mathrm{H}_{74}$ concentration, similar to the static interfacial tension, although polarization is more sensitive than $\theta \mathrm{c}$. The increase in polarization is due to the increase in liquid-liquid interactions that accompanies the increase in paraffinic crystals and aggregates in the liquid phase. The higher sensitivity of polarization over $\theta \mathrm{c}$ was reported before $^{17}$ for monoethylene glycol flowing on polyethylene surfaces with different branching degrees.

For HDPE, the dynamic interfacial tension exhibited a behavior opposite to the static interfacial tension. As the $n-\mathrm{C}_{36} \mathrm{H}_{74}$ concentration increased, the interaction with the walls also increased, showing that the addition of the normal paraffin causes the fluid to have progressively stronger interactions with the HDPE surface under flow. This may be attributed to the $n-\mathrm{C}_{36} \mathrm{H}_{74}$ linear paraffin alkyl chains being stretched by the flow.

Both PP and HDPE have a medium degree of crystallinity, and due to their ASTM preparation method (static melting of their pellets and posterior cooling, under high pressure), their orientation is not well defined. The chain length distribution is wide for both polymers. As PP is a branched polymer, it should have more methyl groups of terminal chains at the surface. Additionally, its branches consist of methyl groups sticking out of the main chain. Thus, it is likely that the PP surface, when compared with the HDPE surface, will have more methyl groups sticking out.

It is known ${ }^{28}$ that, during crystallization, there is segregation between branched molecular sequences and the linear ones due to their different interfacial geometries. Analogous to a growth front of polyethylene, ${ }^{29}$ the existence of methyl groups predominantly in the PP surface, when compared with HDPE, inhibits the interaction with the linear paraffinic alkane content of crude oil. HDPE, on the other hand, has very low number of branches per 1000 carbon atoms and the interaction with stretched linear $n-\mathrm{C}_{36} \mathrm{H}_{74}$ alkane chains is favored, giving rise to more interaction sites per unit area. 
Adding to this, the flow reported here was in one direction and at quite high rate, which, as reported before, ${ }^{21-23}$ induced very high intermolecular alignment. These conditions are different from those of static epitaxial growth, ${ }^{30}$ of dipping/ undipping systems, ${ }^{31}$ and of circular flows, ${ }^{16}$ as the molecules were preferentially orientated in one direction by the liquid flow.

Thus, the long alkyl chains within the liquid, aligned by the flow, have a stronger interaction with the linear chains of HDPE than with the methyl branched PP.

The average polarization over all temperatures and concentrations was lower for PP than for HDPE, showing that, for the conditions studied here, PP has, on average, greater interaction with the crude oil, in accordance with previous observations. ${ }^{4}$

For both surfaces, there was a region with high polarization at $60{ }^{\circ} \mathrm{C}$, at low $n$-paraffin concentrations for HDPE and at high $n$-paraffin concentrations for PP. This points to specific conditions where the dynamic interfacial tension was lowest and the liquid-liquid interactions were strongest. The main characteristics of these two conditions are the high flow rate, which aligns along the flow of any molecular species present within the fluid, combined with the weaker interaction of the $n-\mathrm{C}_{36} \mathrm{H}_{74}$ paraffin with the PP surface relative to the HDPE surface. By differential scanning calorimetry, it was possible to identify the presence of paraffins with ca. 28 carbon atoms that have melting points around $60{ }^{\circ} \mathrm{C}$. Further investigation is needed to elucidate this effect.

\section{Conclusions}

The interfacial interaction between polymer walls and crude oil was investigated at 50,60 , and $70{ }^{\circ} \mathrm{C}$, and at four different concentrations of $n-\mathrm{C}_{36} \mathrm{H}_{74}$, which has a melting point just slightly higher than the temperatures of this study.

The average over all the temperatures and $n$-paraffin concentrations showed that PP has stronger interfacial interactions, both statically and dynamically. Nevertheless, the HDPE has higher interaction with the dissolved $n$-paraffin than the methyl branched PP.

The increase in the static interaction with temperature, regardless of wall chemical constitution, may be due to a decrease in the amount of paraffinic crystals, which decreases the liquid-liquid interaction and increases the wettability of polymer surfaces.

For PP, raising the temperature increased both the static and the dynamic interactions. Thus, increasing the content of dissolved paraffins with chains lengths below 36 carbons caused an increase in the interaction with the methyl branched surface. An increase in $n-\mathrm{C}_{36} \mathrm{H}_{74}$ paraffin concentration decreased both the dynamic and static interactions with the PP surface, showing a progressive tendency of $n-\mathrm{C}_{36} \mathrm{H}_{74}$ to increase the liquid-liquid interaction at the expense of interfacial interaction. This may be attributed to methyl group hindrance of interaction between the PP main chain carbons and longer paraffins on the PP surface. This means that PP is more suitable for linings, pipelines, and ducts that pump crude oils with a high content of linear paraffinic molecules with more than 36 carbon atoms, since the interaction with the walls decreases and should reduce the yield of paraffinic deposits.

For HDPE, the static interaction has very low sensitivity to the $n$-paraffin concentration and steadily increases with temperature. The dynamic interaction, on the other hand, is quite sensitive to the $n$-paraffin concentration. As a general rule, dynamic interaction decreases with temperature, showing an increase in the liquid-liquid interaction as the melted crude paraffinic oil content with less than 32 carbons increases. As the $n-\mathrm{C}_{36} \mathrm{H}_{74}$ paraffin concentration increases, the dynamic interaction with the surface increases. This may be due to both the alignment of the $n$-paraffin chain with the flow and the similar molecular geometry of the $n$-paraffin and the linear polymer surface, which increases the number of sites available for interaction. This means that HDPE is more suitable for linings, ducts, and pipelines that pump crude oils with smaller paraffinic chains.

Acknowledgment. We acknowledge the Conselho Nacional de Desenvolvimento Científico e Tecnológico (CNPq, Brazil) for partial grant support for this work. We acknowledge Politeno for the polymer samples and Eng. Lucas J. D. Britto for their characterization. A.M.V.L. acknowledges an undergraduate research fellowship from CAPES. C.M.Q. acknowledges a senior research scholarship from CNPq.

\section{References and Notes}

(1) Boot, J. C.; Toropova, I. L.; Javadi, A. A. Int. J. Solids Struct 2003, 40 (26), 7299-7314.

(2) Slack, M. Mater. Perform. 1992, 31 (3), 49-52.

(3) Attou, A.; Benamar, A.; Inglebert, G. Mec. Indust. Mater. 1997, 50 (3), 109-111.

(4) Quintella, C. M.; Musse, A. P. S.; Castro, M. T. P. O.; Scaiano, J. C.; Mikelsons, L.; Watanabe, Y. N. Energy Fuels 2006, in press, DOI: $10.1021 /$ ef050267p

(5) Cosulchi, A.; Garciafigueroa, E.; García-Bórquez, A.; Reguera, E.; Yee-Madeira, H.; Lara, V. H.; Bosch, P. Fuel 2001, 80, 1963-1968.

(6) Ajienka, J. A.; Ikoku, C. U. J. Pet. Sci. Eng. 1995, 13, 87-94.

(7) Andreatta, G.; Gonçalves, C. C.; Buffin, G.; Bostrom, N.; Quintella, C. M.; Arteagalarios, F.; Pérez, A.; Mullins, O. C. Energy Fuels 2005, 19 (4), $1282-1289$.

(8) Rocha, N. O.; Gonzalez, G.; Vaitsman, D. S. Quim. Nova 1998, $12(1), 11$

(9) Rademeyer, M.; Dorset, D. L. J. Phys. Chem. B 2001, 105 (22), $5139-5143$

(10) Adamson, A W.; Gast, A. P. Physical Chemistry of Surfaces; John Wiley \& Sons Inc.: New York, 1997.

(11) Extrand, C. W. J. Colloid Interface Sci. 2002, 248, 136-142.

(12) Quintella, C. M.; Gonçalves, C. C.; Castro, M. T. P. O.; Pepe, I.; Musse, A. P. S.; Lima, A. M. V. J. Phys. Chem. B 2003, 107 (33), 8511.

(13) Quintella, C. M.; Gonçalves, C. C.; Pepe, I.; Lima, A. M. V.; Musse, A. P. S. J. Braz. Chem. Soc. 2001, 12, 780-786 (available online, in English, at http://jbcs.sbq.org.br/jbcs/2001/vol12_n6/14.pdf).

(14) Suda, H.; Yamada, S. Langmuir 2003, 19, 529

(15) Quintella, C. M.; Lima, Â. M. V.; Gonçalves, C. C.; Watanabe, Y. N.; Schreiner, M. A.; Mammana, A. P.; Pepe, I.; Pizzo, A. M. J. Colloid Interface Sci. 2003, 262 (1), 221-226.

(16) Zhang, X.; Tian, J.; Wang, L.; Zhou, Z. J. Pet. Sci. Eng. 2002, 36 , $87-95$.

(17) Quintella, C. M.; Musse, A. P. S.; Castro, M. T. P. O.; Gonçalves, C. C.; Watanabe, Y. N. J. Colloid Interface Sci. 2005, 281, 201-208.

(18) Quintella, C. M.; Lima, Â. M. V.; Mammana, A. P.; Schreiner, M. A.; Pepe, I.; Watanabe, Y. N. J. Colloid Interface Sci. 2004, 271, 201205.

(19) Quintella, C. M.; Castro, M. T. P. O. Patent No. MU8302649-5 INPI/BA/Brazil, 2003.

(20) Feofilov, P. P. The Physical Basis of Polarized Emission; Consultants Bureau: New York, 1961.

(21) Kenyon, A. J.; McCaffery, A. J.; Quintella, C. M. Mol. Phys. 1991 $72,965$.

(22) Kenyon, A. J.; McCaffery, A. J.; Quintella, C. M.; Winkel, J. F. Mol. Phys. 1991, 74, 871.

(23) Bain, A. J.; Chandna, P.; Butcher, G. Chem. Phys. Lett. 1996, 260, 441-446.

(24) Quintella, C. M.; Gonçalves, C. C.; Musse, A. P. S.; McCaffery, A. J. Exp. Fluids 2003, 35 (1), 41.

(25) Quintella, C. M.; Musse, A. P. S.; Gonçalves, C. C. J. Phys. Chem. B 2004, 108 (8), 2751-2755.

(26) Castro, M. T. P. O.; Quintella, C. M. Quim. Nova 2005, 28 (2), $337-337$.

(27) Quintella, C. M.; Gonçalves, C. C.; Pepe, I.; Lima, A. M. V.; Musse, A. P. S. J. Autom. Methods Manage. Chem. 2002, 24, 31-39 (available online in English at http://taylorandfrancis.metapress.com).

(28) Hosier, I. L.; Bassett, D. C. Polym. J. 1999, 31 (9), 772-777.

(29) Janimak, J. J.; Bassett, D. C. Polym. 1999, 40 (2), 459-468.

(30) Kawagushi, A.; Patermann, J. J. Polym. Sci. 1985, 23 (12), 26232627.

(31) Bourgoin, J. P.; Doublet, F.; Palacin, S.; Vandevyver, M. Langmuir 1996, 12 (26), 6473-6479. 